\title{
Reduced-Order Modelling of parameterized transient flows in closed-loop systems
}

\author{
Péter German ${ }^{1 *}$ Mauricio Tano ${ }^{1}$, Jean C. Ragusa ${ }^{1}$ and Carlo Fiorina ${ }^{2}$ \\ ${ }^{1}$ Department of Nuclear Engineering, Texas A\&M University \\ 3133 TAMU, 423 Spence St, College Station, TX-77843, USA
}

${ }^{2}$ Laboratory for Reactor Physics and Systems Behaviour, École Polytechnique Fédérale de Lausanne PH D2 435, Station 3, CH-1015 Lausanne, Switzerland

peter.german@tamu.edu,mtano@tamu.edu,jean.ragusa@tamu.edu, carlo.fiorina@epfl.ch

\begin{abstract}
In this paper, two Galerkin projection based reduced basis approaches are investigated for the reduced-order modeling of parameterized incompressible Navier-Stokes equations for laminar transient flows. The first approach solves only the reduced momentum equation with additional, physics-based approximations for the dynamics of the pressure field. On the other hand, the second approach solves both the reduced momentum and continuity equations. The reduced bases for the velocity and pressure fields are generated using the method of snapshots combined with Proper Orthogonal Decomposition (POD) for data compression. To remedy the stability issues of the two-equation model, the reduced basis of the velocity is enriched with supremizer functions. Both reduced-order modeling approaches have been implemented in GeN-Foam, an OpenFOAM-based multi-physics solver. A numerical example is presented using a two-dimensional axisymmetric model of the Molten Salt Fast Reactor (MSFR) and the dynamic viscosity as the uncertain parameter. The results indicate that the one-equation model is slightly more accurate in terms of velocity, while the two-equation model, built with the same amount of modes for the velocity, is far more accurate in terms of pressure. The speed-up factors for the reduced-order models are 3060 for the one-equation model and 2410 for the two-equation model.
\end{abstract}

KEYWORDS: Reduced-Order Modeling, ROM, Parametric Model Order Reduction, OpenFOAM

\section{INTRODUCTION}

The simulation of complex phenomena requires, in many cases, the solution of a system of partial differential equations in a discretized form. In such systems, the number of unknowns, or degrees of freedom, is potentially large and, consequently, obtaining a solution is computationally expensive. Simulation of transient fluid flows can serve as a good example for such complex problems.

${ }^{*}$ Corresponding author 
The use of these expensive Full-Order Models (FOMs) is often challenging for applications that entail a large number of simulations with variability in input parameters, e.g., design optimization or uncertainty quantification. In these situations, the construction of Reduced-Order Models (ROMs) can decrease the computational burden by reducing the number of unknowns, with often modest and controllable sacrifice from the side of accuracy. This paper investigates the applicability of two projection-based reduced-order modeling approaches to simulate parameterized laminar transient fluid flows. Both of the techniques focus on deriving ROMs from FOMs with finite-volume spatial discretization. The first approach used in this paper was published in [1] and uses a one-equation technique with physics-based approximations for the pressure field. The second approach, on the other hand, yields a two-equation ROM [2]. Both proved to be accurate methods for inlet-outlet problems as shown in [3-5]. These methods are adopted in this work for another typical problem class in the nuclear industry, namely closed-loop systems with volumetric momentum sources. The applicability of the two reduced-order modeling techniques is presented using a pump start-stop transient scenario with a 2D axisymmetric model of the Molten Salt Fast Reactor (MSFR) [6]. For full-order simulations, the OpenFOAM [7] based finite volume multi-physics solver GeN-Foam [8] is used. All the routines used for the reduced-order modeling part have been implemented within GeN-Foam as well. The rest of the paper is organized as follows: in Section 2 we review the mathematical models used to describe fluid flows in laminar regimes; in Section 3 we provide the derivations for the corresponding ROMs, while Section 4 provides the numerical example for the MSFR.

\section{FULL-ORDER GOVERNING EQUATIONS}

Incompressible fluid flows in the laminar regime can be described accurately using the NavierStokes equations [9]; these express momentum and mass conservation in the following form:

$$
\begin{aligned}
\frac{\partial \rho \boldsymbol{u}}{\partial t}+\boldsymbol{\nabla} \cdot(\rho \boldsymbol{u} \otimes \boldsymbol{u}) & =\boldsymbol{\nabla} \cdot\left(\eta\left[\boldsymbol{\nabla} \boldsymbol{u}+(\boldsymbol{\nabla} \boldsymbol{u})^{T}\right]\right)-\boldsymbol{\nabla} p+\boldsymbol{S} & & \boldsymbol{r} \in \Omega, \\
\boldsymbol{\nabla} \cdot \rho \boldsymbol{u} & =0 & & \boldsymbol{r} \in \Omega,
\end{aligned}
$$

where $\boldsymbol{u}$ denotes the velocity vector, $p$ the pressure, $\eta$ the molecular dynamic viscosity, and $\rho$ the density within the computational domain $\Omega$ with boundary $\partial \Omega$. In addition, $\boldsymbol{S}$ incorporates all additional volumetric momentum sources or sinks. In this paper we consider incompressible fluids only, which means that the density in the equations above is constant. Furthermore, the dynamic viscosity is assumed to be constant as well. The conservation equations are supplemented with appropriate initial and boundary conditions. For a closed loop system, the boundary conditions are given in the following form:

$$
\begin{aligned}
\boldsymbol{u} & =\mathbf{0} & & \boldsymbol{r} \in \partial \Omega, \\
\nabla p \cdot \boldsymbol{n} & =0 & & \boldsymbol{r} \in \partial \Omega .
\end{aligned}
$$

and the initial velocity and pressure fields, $\boldsymbol{u}(0, \boldsymbol{r})$ and $p(0, \boldsymbol{r})$ are given. These equations are then discretized in space and time. In this paper we use a finite-volume treatment in space and an implicit-Euler discretization in time. Lastly, it must be mentioned that input parameters in the Full-Order Model (FOM), such as molecular dynamic viscosity, fluid density, or parameters in the source term $S$, can be uncertain with different distributions, yielding a parameterized problem. Henceforth, we include all of these uncertain parameters into a parameter vector denoted by $\boldsymbol{\mu}=$ $[\eta, \rho, \ldots]^{T}$. 


\section{REDUCED GOVERNING EQUATIONS}

In this section, two approaches for obtaining ROMs for laminar incompressible Navier-Stokes equations are described. The first one, described in [2,5] for a finite volume setting, reduces Eqs. (1)-(2), thus it is referred to as two-equation ROM. The second approach, first described in [1] and often referred to as POD-FV-ROM, on the other hand, reduces Eq. (1) only by supplying approximations for the pressure field. Therefore, it is referred to as one-equation ROM in this paper. The method of snapshots combined with Proper Orthogonal Decomposition (POD) [10] is used to obtained the basis functions, often called modes, of the reduced subspaces for both ROM types. In principle, the generation of these ROMs comprises two major phases, an offline phase, that has to be carried out only once, at the beginning, and an online step that is performed every time the ROM is to be solved.

\subsection{Offline stage}

The offline phase begins with gathering information about the time and parameter dependent solution manifolds, meaning that the FOM is exercised to obtain $N_{s}$ snapshots of the system at different time instances or parameter values. In case of finite volume discretization, a snapshot can be considered to be a vector containing the value of a scalar (pressure) or vector (velocity) at the center of each cell. Assuming that domain $\Omega$ is partitioned into $N$ cells, the size of one snapshot is $N$ for the pressure and $3 N$ for the velocity in 3D. These snapshots are collected in snapshot matrices

$$
\begin{array}{r}
\boldsymbol{R}_{u}=\left[\boldsymbol{u}_{1}, \ldots, \boldsymbol{u}_{N_{s}}\right] \\
\boldsymbol{R}_{p}=\left[p_{1}, \ldots, p_{N_{s}}\right]
\end{array}
$$

for velocity and pressure. Both reduction approaches use these snapshot matrices to obtain the basis vectors (or modes) of the reduced subspaces. However, the way the bases are obtained is slightly different for the two methods. For the two-equation ROM, it is done by solving the following two eigenvalue problems as a first step

$$
\begin{aligned}
& \mathcal{C}^{u} \boldsymbol{V}^{u}=\boldsymbol{\Lambda}^{u} \boldsymbol{V}^{u}, \\
& \mathcal{C}^{p} \boldsymbol{V}^{p}=\boldsymbol{\Lambda}^{p} \boldsymbol{V}^{p},
\end{aligned}
$$

where $\mathcal{C}^{u}$ and $\mathcal{C}^{p}$ (both of size $N_{s} \times N_{s}$ ) are the correlation matrices of the velocity and pressure snapshots with respect to an analytic $L^{2}$ norm. Using the resulting eigenvectors and eigenvalues, basis vectors $\phi_{i}^{u}$ and $\phi_{i}^{p}$ for the velocity and pressure subspaces are constructed with the help of the snapshots as follows:

$$
\begin{aligned}
& \phi_{i}^{u}=\frac{1}{\sqrt{\lambda_{i}^{u}}} \sum_{k=0}^{N_{s}} \boldsymbol{u}_{k} \boldsymbol{V}_{i, k}^{u}, \\
& \phi_{i}^{p}=\frac{1}{\sqrt{\lambda_{i}^{p}}} \sum_{k=0}^{N_{s}} p_{k} \boldsymbol{V}_{i, k}^{p} .
\end{aligned}
$$

where $\lambda_{i}^{u}$ and $\lambda_{i}^{p}$ are the eigenvalues of the correlation matrices, located on the diagonals of $\Lambda^{u}$ and $\Lambda^{p}$. In most cases, the number of relevant basis vectors is much smaller than the number of snapshots, $r_{u} \ll N_{s}$ for velocity and $r_{p} \ll N_{s}$ for pressure. This might allow further reduction in 
the dimension of the subspaces. The importance of each basis function compared to the others can be determined by assessing the decay in the eigenvalues of the correlation matrices.

In case of the one-equation method, the reduced bases are obtained slightly differently. Only Eq. (5) is solved alone, and the modes are given by

$$
\begin{aligned}
& \phi_{i}^{u}=\frac{1}{\sqrt{\lambda_{i}^{u}}} \sum_{k=0}^{N_{s}} \boldsymbol{u}_{k} \boldsymbol{V}_{i, k}^{u}, \\
& \phi_{i}^{p}=\frac{1}{\sqrt{\lambda_{i}^{u}}} \sum_{k=0}^{N_{s}} p_{k} \boldsymbol{V}_{i, k}^{u} .
\end{aligned}
$$

where the pressure modes are constructed in a way that they are required to follow the same dynamics as the velocity modes. This approximation was first introduced in [1] and it has been experimentally demonstrated to yield accurate ROMs for practical inlet-outlet applications [3].

Once the basis vectors of the reduced subspaces are available, the solution of the full-order equations is approximated as the linear combinations of these modes

$$
\begin{aligned}
& \boldsymbol{u}(\boldsymbol{r}, t ; \mu) \approx \sum_{i=0}^{r_{u}} \phi_{i}^{u}(\boldsymbol{r}) c_{i}^{u}(t, \mu), \\
& p(\boldsymbol{r}, t ; \mu) \approx \sum_{i=0}^{r_{p}} \phi_{i}^{p}(\boldsymbol{r}) c_{i}^{p}(t, \mu),
\end{aligned}
$$

for the two-equation ROM, and

$$
\begin{aligned}
& \boldsymbol{u}(\boldsymbol{r}, t ; \mu) \approx \sum_{i=0}^{r_{u}} \phi_{i}^{u}(\boldsymbol{r}) c_{i}^{u}(t, \mu), \\
& p(\boldsymbol{r}, t ; \mu) \approx \sum_{i=0}^{r_{u}} \phi_{i}^{p}(\boldsymbol{r}) c_{i}^{u}(t, \mu),
\end{aligned}
$$

for the one-equation ROM. Here, $c_{i}^{u}(t, \mu)$ and $c_{i}^{p}(t, \mu)$ denote the coordinates of the full order fields in the reduced subspaces. Finally, the full-order equations are projected onto the reduced subspace to get the reduced-order systems. In this work, a Galerkin projection is used, however, as discussed in [11], Petrov-Galerkin projection presents a powerful alternative as well. The reduced laminar fluid equations with the two-equation method become

$$
\begin{aligned}
M \dot{\boldsymbol{c}}^{u}+\boldsymbol{c}^{u, T} \boldsymbol{C} \boldsymbol{c}^{u}-\eta D \boldsymbol{c}^{u}+P \boldsymbol{c}^{p} & =\boldsymbol{S}_{r} \\
G \boldsymbol{c}^{u} & =0
\end{aligned}
$$

where the reduced operators are

$$
\begin{aligned}
M_{i, j} & =\left\langle\phi_{i}^{u}, \rho \phi_{j}^{u}\right\rangle_{\Omega} & \boldsymbol{C}_{i, j, k} & =\left\langle\phi_{j}^{u}, \boldsymbol{\nabla} \cdot\left(\rho \phi_{i}^{u}\right.\right. \\
D_{i, j} & =\left\langle\phi_{i}^{u}, \boldsymbol{\nabla} \cdot\left[\boldsymbol{\nabla} \phi_{j}^{u}+\left(\boldsymbol{\nabla} \phi_{j}^{u}\right)^{T}\right]\right\rangle_{\Omega} & P_{i, j} & =\left\langle\phi_{i}^{u}, \boldsymbol{\nabla} \phi_{j}^{p}\right\rangle_{\Omega} \\
G_{i, j} & =\left\langle\phi_{i}^{u}, \boldsymbol{\nabla} \cdot \phi_{j}^{u}\right\rangle_{\Omega} & \boldsymbol{S}_{r, i} & =\left\langle\phi_{i}^{u}, \boldsymbol{S}\right\rangle_{\Omega} .
\end{aligned}
$$


It is important to note that these reduced matrices have to be computed only once, they can be stored, and every ROM solve only involves multiplication by constant and summation of small dense matrices, which is of low computational cost.

Unfortunately, even simple two-equation models have stability problems at reduced-order level. To tackle this, a supremizer stabilization has been adopted [12]. The essence of this method is that additional stabilizing, so called supremizer basis functions are mixed to the velocity modes. For more information the reader is referred to $[2,5,12]$.

In case of the one-equation ROM, the following, considerably simpler, system is solved:

$$
M \dot{\boldsymbol{c}}^{u}+\boldsymbol{c}^{u, T} \boldsymbol{C} \boldsymbol{c}^{u}-\eta D \boldsymbol{c}^{u}+P \boldsymbol{c}^{u}=\boldsymbol{S}_{r} .
$$

A noteworthy advantage of this method is that, based on $[1,3]$, it does not exhibit stability issues, unlike its two-equation counterpart.

\subsection{Online stage}

Following the computationally expensive offline phase, the online phase consists of only two, relatively cheap steps. First, the reduced equations are assembled from the small matrices precomputed in the offline procedure, then the reduced algebraic equation system is solved and the approximate full-order solution is reconstructed using Eqs. (11)-(14). In this study, a simple fixed-point iteration is used to solve the reduced nonlinear equations. To compare the results from the ROMs to those of the FOM we adopt the following error metrics:

$$
\begin{aligned}
\delta \boldsymbol{u}(t ; \mu) & =\frac{\left\|\boldsymbol{u}_{F O M}(\boldsymbol{r}, t ; \mu)-\boldsymbol{u}_{R O M}(\boldsymbol{r}, t ; \mu)\right\|_{L^{2}}}{\left\|\boldsymbol{u}_{F O M}(\boldsymbol{r}, t ; \mu)\right\|_{L^{2}}}, \\
\delta p(t ; \mu) & =\frac{\left\|p_{F O M}(\boldsymbol{r}, t ; \mu)-p_{R O M}(\boldsymbol{r}, t ; \mu)\right\|_{L^{2}}}{\left\|p_{F O M}(\boldsymbol{r}, t ; \mu)\right\|_{L^{2}}}
\end{aligned}
$$

\section{NUMERICAL EXAMPLE}

For demonstration purposes, a 2D axisymmetric model of the Molten Salt Fast Reactor (MSFR) has been created using the available information in [6] and [13]. The geometry of the model is presented in Figure 1 with the red area showing the location of the pump which is treated as a volumetric momentum source. The hexahedral mesh consists of 16,067 cells meaning that the number of unknowns in the full-order model is 48,021. For all full-order runs the density of the fluid is $4125 \frac{\mathrm{kg}}{\mathrm{m}^{3}}$, while the dynamic viscosity is considered to be an uncertain parameter varying in the range of $[0.1,0.5] \mathrm{Pa} \cdot \mathrm{s}$ with a uniform distribution. For this work the following transient scenario has been chosen (see also Figure 2):

1. $0 s \leq t \leq 100 \mathrm{~s}$ : The pump is started and linearly ramped up from $|\boldsymbol{S}|=0 \frac{\mathrm{N}}{\mathrm{m}^{3}}$ to $2.5 \frac{\mathrm{N}}{\mathrm{m}^{3}}$

2. $100 s<t \leq 600 \mathrm{~s}$ : The pumping power stays constant at $|\boldsymbol{S}|=2.5 \frac{\mathrm{N}}{\mathrm{m}^{3}}$

3. $600 \mathrm{~s}<t \leq 700 \mathrm{~s}$ : The pumping power is decreased linearly from $|\boldsymbol{S}|=2.5 \frac{\mathrm{N}}{\mathrm{m}^{3}}$ to $0 \frac{\mathrm{N}}{\mathrm{m}^{3}}$

4. $700 \mathrm{~s}<t \leq 1000 \mathrm{~s}$ : The pump stays shut down, while the residual inertia of the fluid slowly dissipates 


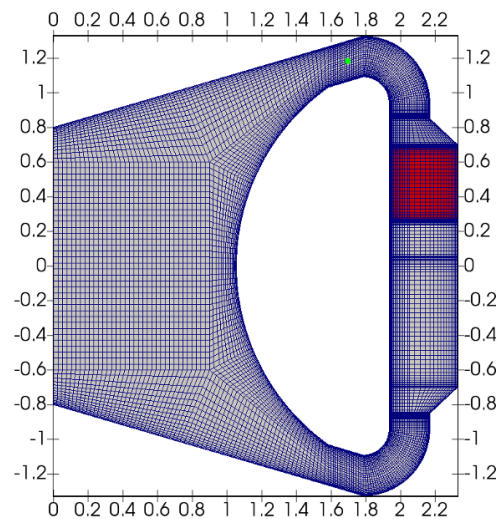

Figure 1: 2D axisymmetric geometry of the MSFR used for the numerical example. (red: pump region, green: probe location, gray: regular flow domain)

At the beginning of the offline phase, five values of the dynamic viscosity are chosen with a uniform spacing between the maximum and minimum values, including the bounds as well. Then the previously described transient scenario is solved with the full-order model for all five values of the viscosity using a time step size of $\Delta t=1 \mathrm{~s}$. Snapshots of the velocity and pressure fields are saved every $10 \mathrm{~s}$ resulting in 500 snapshots (100 snapshot per scenario) for each solution field altogether.

Using the information gained from the snapshots, two reduced-order models have been built. A one-equation ROM using 24 basis functions for the velocity and pressure, and a two-equation ROM using 24 basis functions for the velocity and 8 basis functions for the pressure and supremizer fields. After the generation of these ROMs, the dynamic viscosity is set to $\eta=0.2 \dot{3} \mathrm{~Pa} \cdot \mathrm{s}$, which is not included in the training values. The two ROMs and the FOM are run using this input parameter and the results are compared. The magnitude of the velocity is evaluated at the inlet of the heat exchanger (green dot in Figure 1) and the results are shown in Figure 2.

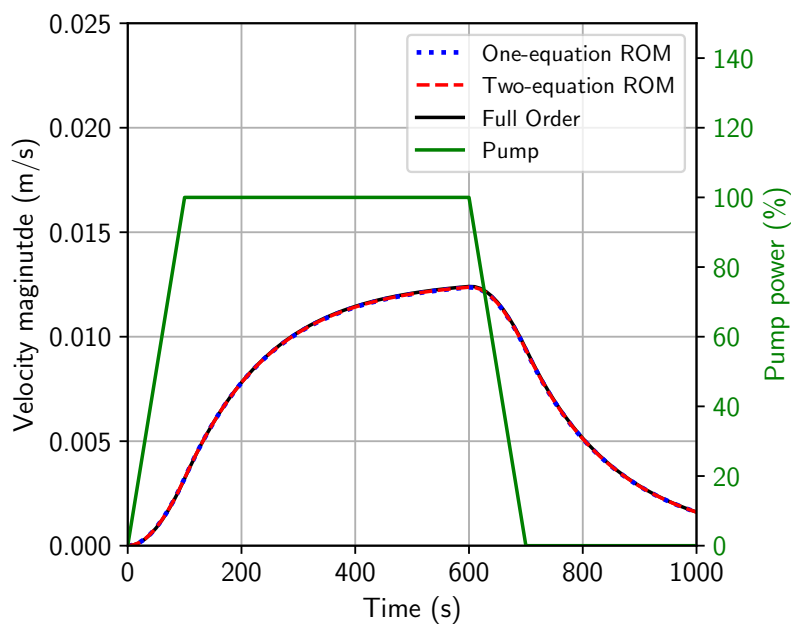

Figure 2: Comparison of the velocity magnitudes at the probe location. 
It is visible that both ROM approaches yield accurate results, however the exact measure of error is difficult to read. For this reason, time dependent error indicators Eq. (18)-(19) are also plotted in Figure 3. To show the applicability of these convoluted ROMs, another line has been plotted showing the achievable accuracy with simple linear interpolation (in the parameter space) using the snapshots directly. It can be observed that besides the first $80 \mathrm{~s}$ of the transient, the relative $L^{2}$ error in the velocity is lower than $1 \%$ for both ROM approaches. It is important to note that the one-equation ROM is slightly more accurate in terms of velocity, however it is far worse in terms of pressure. The accuracy of the pressure for the two-equation ROM can be increased by the inclusion of additional pressure and supremizer modes if necessary. It is also visible, that even though the linear interpolation yields accurate results in terms of pressure, it is consistently outperformed by the ROMs in case of the velocity after the first 100 seconds of the simulation.
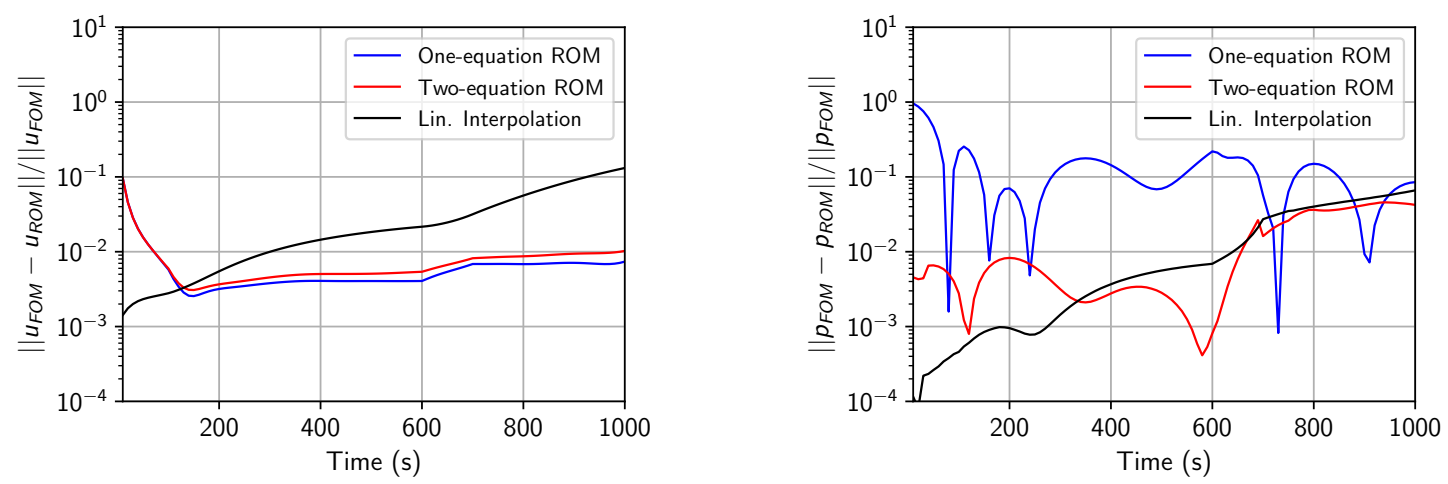

Figure 3: Velocity and pressure errors as function of time for the one- and two-equation ROMs.

The observed speed-up factor is approximately 3060 for the one-equation ROM and 2410 for its two-equation counterpart. This implies that if more than five runs are necessary for the optimization of the dynamic viscosity or related uncertainty quantification, and the presented relative errors are acceptable, it is worth creating and using ROMs for subsequent runs.

\section{CONCLUSIONS}

The applicability of two reduced-order modeling approaches for parameterized transient laminar fluid flows in closed-loop systems has been studied. A transient scenario including pump start-up and shut down in the MSFR is investigated with the dynamic viscosity as uncertain parameter. The results indicate that in cases when the accurate reconstruction of the pressure field is not necessary, the one-equation ROM is better, since it is simpler and inherently faster to solve while providing approximately the same level of accuracy for the velocity field. However, when knowing accurately the pressure is essential, the two-equation ROM approach is recommended because it can achieve errors two orders of magnitude lower than those of the one-equation ROM. The ROMs yield speedup factors of approximately 3060 in the one equation case and 2410 in the two-equation, meaning that creating ROMs can be beneficial if more than five runs are necessary and the error levels attained are acceptable. 


\section{ACKNOWLEDGEMENTS}

This material is based upon work supported under an NEUP-IRP Award of the Department of Energy, Office of Nuclear Energy (contract reference DE-NE0008651).

\section{REFERENCES}

[1] S. Lorenzi, A. Cammi, L. Luzzi, and G. Rozza. "POD-Galerkin method for finite volume approximation of Navier-Stokes and RANS equations." Computer Methods in Applied Mechanics and Engineering, volume 311, pp. 151-179 (2016).

[2] G. Stabile and G. Rozza. "Finite volume POD-Galerkin stabilised reduced order methods for the parametrised incompressible Navier-Stokes equations." Computers \& Fluids, volume 173, pp. 273-284 (2018).

[3] S. Lorenzi, A. Cammi, L. Luzzi, and G. Rozza. "A reduced order model for investigating the dynamics of the Gen-IV LFR coolant pool." Applied Mathematical Modelling, volume 46, pp. 263-284 (2017).

[4] S. Hijazi, S. Ali, G. Stabile, F. Ballarin, and G. Rozza. "The effort of increasing Reynolds number in projection-based reduced order methods: from laminar to turbulent flows." arXiv preprint arXiv: 180711370 (2018).

[5] S. Hijazi, G. Stabile, A. Mola, and G. Rozza. "Data-driven POD-Galerkin reduced order model for turbulent flows." arXiv preprint arXiv: 190709909 (2019).

[6] M. Aufiero, A. Cammi, O. Geoffroy, M. Losa, L. Luzzi, M. E. Ricotti, and H. Rouch. "Development of an OpenFOAM model for the Molten Salt Fast Reactor transient analysis." Chemical Engineering Science, volume 111, pp. 390-401 (2014).

[7] H. Jasak, A. Jemcov, Z. Tukovic, et al. "OpenFOAM: A C++ library for complex physics simulations." In International workshop on coupled methods in numerical dynamics, volume 1000, pp. 1-20. IUC Dubrovnik Croatia (2007).

[8] C. Fiorina, I. Clifford, M. Aufiero, and K. Mikityuk. "GeN-Foam: a novel OpenFOAMß based multi-physics solver for 2D/3D transient analysis of nuclear reactors." Nuclear Engineering and Design, volume 294, pp. 24-37 (2015).

[9] R. Temam. Navier-Stokes equations: theory and numerical analysis. AMS Chelsea Publishing, Bloomington IN, USA (2001).

[10] K. Willcox and J. Peraire. "Balanced model reduction via the proper orthogonal decomposition." AIAA journal, volume 40(11), pp. 2323-2330 (2002).

[11] K. Carlberg, M. Barone, and H. Antil. “Galerkin v. least-squares Petrov-Galerkin projection in nonlinear model reduction." Journal of Computational Physics, volume 330, pp. 693-734 (2017).

[12] F. Ballarin, A. Manzoni, A. Quarteroni, and G. Rozza. "Supremizer stabilization of PODGalerkin approximation of parametrized steady incompressible Navier-Stokes equations." International Journal for Numerical Methods in Engineering, volume 102(5), pp. 1136-1161 (2015).

[13] C. Fiorina, D. Lathouwers, M. Aufiero, A. Cammi, C. Guerrieri, J. L. Kloosterman, L. Luzzi, and M. E. Ricotti. "Modelling and analysis of the MSFR transient behaviour." Annals of Nuclear Energy, volume 64, pp. 485-498 (2014). 